\title{
Liver surgery has become simpler
}

Liver resections were once formidable operations. Mobilization of the liver often resulted in twisting or compression of the retrohepatic inferior vena cava leading to blockade of the venous return. The liver parenchyma was sometimes torn when trying to mobilize the right lobe of the liver, particularly in bulky tumours. The division of the short liver ligaments sometimes resulted in bleeding or injury to the diaphragm. Liver parenchyma transection was always a source of persistent heavy bleeding from the raw liver stump. The dissection of major hepatic veins was much feared, owing to the risk of injury and ensuing air embolism. Dissection at the hilum was considered dangerous because it was associated with a risk of postoperative bile leaks. This prompted liver surgeons to divide portal pedicles inside the liver during parenchymal transection rather than outside the liver before transection. The use of large drains was then recommended. In his first description of liver trisegmentectomies in the early 1970s, Starzl, a pioneer in liver surgery, advocated the use of prolonged packing at the end of resections to prevent postoperative bleeding.

Postoperative follow-up was plagued by severe complications including bleeding, bile fistula and subphrenic abscesses. For all of these reasons, the intraoperative course was difficult and required invasive monitoring of the patient including measurement of arterial blood pressure and central venous pressure by indwelling catheters, transfusion of packed red cells, fresh frozen plasma, platelets or antifibrinolytic agents and often the administration of vasoactive drugs. Postoperative hospitalization in a surgical intensive care unit (ICU) was mandatory. Prolonged artificial ventilation was recommended. Postoperative complications were frequent and the death rate high. Planning liver surgery was a source of nervousness and excitement in the operating room for both surgeons and anaesthesiologists. It was considered a major operation. The procedure was most

Correspondence to: Dominique Franco, Hôpital Antoine Béclère, F-92141 Clamart Cédex, France. E-mail: dominique.franco@abc.ap-hop-paris.fr; Tel: +33 1453743 48; Fax: +33145374978

Accepted for publication July 2002 EJA 909 dangerous when the liver parenchyma was fibrotic or congested by bile.

Fortunately things have changed over the years and liver resections have become much less risky. This has resulted from improvements in every step of liver surgery, including accurate preoperative analysis and careful planning of the surgical procedure. Computed tomographic scanning and magnetic resonance imaging provide information on liver anatomy and make it possible to predict most intraoperative problems [1]. It is possible to see variations in portal, arterial and bile duct anatomy, and to visualize relationships between the hepatic veins, vascular system, diaphragm and the tumour, thus leading to a better surgical management. Three-dimensional reconstruction of the liver may soon make virtual transection possible and thus further advance the operative field [2].

The surgical approach to liver resection has also been greatly simplified. The devastating thoracoabdominal approach is now only used in a very small number of patients with tumours invading the cavohepatic junction. A long subcostal abdominal incision is an excellent approach for most resections, providing enough space to mobilize the right or left lobe of the liver without compressing or twisting the inferior vena cava. In patients with large tumours of the right lobe, in whom mobilization of the right lobe can lead to complications, the so-called 'anterior approach' makes complete parenchymal transection possible before mobilizing the enlarged right lobe [3].

One of the major advances in liver surgery is the prevention of intraoperative bleeding. This includes several points:

- Maintaining a low central venous pressure by avoiding the massive intravenous fluid infusion that used to be undertaken at the beginning of anaesthesia for liver surgery [4].

- Control and division of major portal pedicles and hepatic veins before parenchymal transection.

- Use of vascular clamping during transection. Portal triad clamping is the most important procedure. It decreases bleeding significantly during transection [5] and is very well tolerated for a long period. It has little effect on systemic haemodynamics [6] 
or liver function [7], particularly if intermittent $[8,9]$. The clamping of the portal triad does not require steady haemodynamic surveillance. Although rupture of the spleen and bacterial translocation have occasionally been reported, these are uncommon events. Total vascular exclusion was once largely advocated, but this is no more efficient and definitely less well tolerated than portal triad clamping [10]. It also requires invasive monitoring and resuscitation procedures. Above all, it has recently been suggested that most liver resections, including those with partial resection of the inferior vena cava or dissection of the cavohepatic junction, can be performed without total vascular exclusion [11].

- Gentle transection of the liver parenchyma by smooth fracture using a small pair of forceps (kellyclasia), making it possible to dissect and to divide electively every tiny vascular or biliary branch. Many new technologies have improved the transection of liver parenchyma, including ultrasonic dissectors, high-pressure water jets and harmonic scalpels. Although these tools may help an experienced liver surgeon, particularly when approaching large hepatic veins or portal pedicles, their superiority over conventional small clamp crushing has not been proven. Bipolar coagulation is one procedure that has led to the biggest improvement in parenchymal transection. It is now used to obstruct almost all pedicles encountered during any type of liver resection, the only exception being the largest branches of major hepatic veins. It avoids the many long and tedious ligations previously required to secure haemostasis and biliary stasis of the hepatic stump. When transection is carefully performed, the hepatic stump does not usually bleed at the end of resection and does not require any complementary procedures. Transient packing of the liver stump by applying gentle hand pressure stops most bleeding and is much better than suturing the fragile liver parenchyma. Biological glue, argon beam coagulation and other new technologies are expensive and not usually useful in treating the liver stump. The quality of the stump is usually such that abdominal drainage is not needed [12].

With all these technical refinements, most liver resections, including standard, major or extended hepatectomies, have become uneventful procedures. The risk of unexpected life-threatening complications during surgery is extremely low. In most cases, less than $500 \mathrm{~mL}$ blood is lost and transfusion is necessary in less than $20 \%$ of patients [13,14]. It can also be predicted by using clinical scores [15]. As the patient's haemodynamics do not usually change during surgery, intraoperative monitoring can be minimal. Peripheral cannulas are sufficient for intraoperative fluid infusion and for the administration of anaesthetic drugs. Following the operation, the patient does not have to be hospitalized in an ICU, but can be simply managed in a conventional surgical ward. When everything goes well in the operating room, the rate of severe postoperative complications is low. There is no need for specific postoperative therapy. Oral feeding is quickly resumed. The postoperative hospitalization period is short. Liver surgery in these patients compares with other types of abdominal surgery. Intra- and postoperative surveillance is dictated only by associated diseases that may require specific surveillance. The operative mortality rate after such resections is virtually zero [16-18]. This represents most liver resections for patients with reasonable sized tumours, even if the liver is fibrotic, and there is no invasion of the diaphragm, major hepatic veins, inferior vena cava or portal pedicle. Even liver resection in patients with cirrhosis follows the same pattern providing that surgery is carefully indicated and patients have normal liver function [19].

Conversely, a few liver resections do result in intraoperative complications, particularly sudden and/or prolonged bleeding and air embolism. However, these can usually be predicted preoperatively. Patients are at risk if they require a large hepatectomy for a large tumour particularly in the right lobe [20], if they have a tumour near the inferior vena cava or cavohepatic junction, or if the major portal branches have been invaded. In addition to liver resection, diaphragmatic excision, vascular repair and total vascular exclusion may be performed. Such technical difficulties can and must be predicted before surgery by careful investigations so that invasive haemodynamic monitoring is planned and everything is prepared in the operative room for a rapid, large-volume transfusion. Postoperative surveillance in a surgical ICU is only necessary in these cases. If unexpected technical difficulties are encountered in a patient scheduled to undergo standard liver resection, the mobilization of the liver should be delayed until monitoring can be started. When performing liver resection, it is important to remember that packing can stop unexpected bleeding, thus making it possible for the anaesthesiologist to adapt the monitoring technique.

In this issue of the Journal (pages 780-788), Lentschener and Ozier advocate the simplification of intra- and postoperative management of liver resection [21]. Simplification of liver surgery and continuous improvements to liver resection justify this attitude. Unexpected intraoperative complications should become the exception.

It is still not clear whether laparoscopy will alter the management of liver resection patients. There 
are strong arguments suggesting that many liver resections will soon be performed by a laparoscopic approach. Early experience suggests that the risk of bleeding is not changed during laparoscopy [22]. Magnification makes it easier to dissect large hepatic veins and to transect the parenchyma. Bleeding from the exposed liver surface may be decreased by pressure from the pneumoperitoneum and the risk of air embolism seems quite low. This technique remains to be explored.

\section{Dominique Franco Hôpital Antoine Béclère Clamart, France}

\section{References}

1. Alfieri S, Carriero C, Caprino P, et al. Avoiding early postoperative complications in liver surgery. A multivariate analysis of 254 patients consecutively observed. Dig Liver Dis 2001; 33: 341-346.

2. Rau HG, Schauer R, Helmberger T, et al. Impact of virtual reality imaging on hepatic liver tumor resection: calculation of risk. Langenbecks Arch Surg 2000; 385: 162-170.

3. Liu CL, Fan ST, Lo CM, Tung-Ping Poon RT, Wong J. Anterior approach for major right hepatic resection for large hepatocellular carcinoma. Ann Surg 2000; 232: 25-31.

4. Melendez JA, Arslan V, Fischer ME, et al. Perioperative outcomes of major hepatic resections under low central venous pressure anesthesia. Blood loss, blood transfusion, and the risk of postoperative renal dysfunction. $J$ Am Coll Surg 1998; 187: 620-625.

5. Man K, Fan ST, Ng IO, Lo CM, Liu CL, Wong J. Prospective evaluation of Pringle maneuver in hepatectomy for liver tumors by a randomized study. Ann Surg 1997; 226: 704-713.

6. Lentschener C, Franco D, Bouaziz H, et al. Haemodynamic changes associated with portal triad clamping are suppressed by prior hepatic pedicle infiltration with lidocaine in humans. BrJ Anaesth 1999; 82: 691-697.

7. Takayama T, Makuuchi M, Kubota K, et al. Randomized comparison of ultrasonic vs clamp transection of the liver. Arch Surg 2001; 136: 922-928.

8. Belghiti J, Noun R, Malafosse R, et al. Continuous versus intermittent portal triad clamping for liver resection: a controlled study. Ann Surg 1999; 229: 369-375.
9. Torzilli G, Makuuchi M, Inoue K. The vascular control in liver resection: revisitation of a controversial issue. Hepatogastroenterology 2002; 49: 28-31.

10. Belghiti J, Noun R, Zante E, Ballet T, Sauvanet A. Portal triad clamping or hepatic vascular exclusion for major liver resection. A controlled study. Ann Surg 1996; 224: $155-161$.

11. Torzilli G, Makuuchi M, Midorikawa Y, et al. Liver resection without total vascular exclusion: hazardous or beneficial? An analysis of our experience. Ann Surg 2001; 233: 167-175.

12. Franco D, Karaa A, Meakins JL, Borgonovo G, Smadja C, Grange D. Hepatectomy without abdominal drainage: results of a prospective study in 61 patients. Ann Surg 1989; 210: 748-751.

13. Lentschener C, Benhamou D, Mercier F, et al. Aprotinin reduces blood loss in patients undergoing elective liver resection. Anesth Analg 1997; 84: 875-881.

14. Jones RM, Moulton CE, Hardy KJ. Control venous pressure and its effects on blood loss during liver resection. Br J Surg 1998; 85: 1058-1060.

15. Mariette D, Smadja C, Naveau S, Borgonovo G, Vons C, Franco D. Preoperative predictors of blood transfusion in liver resection for tumor. Am J Surg 1997; 173: 275-279.

16. Franco D, Smadja C, Meakins JL, Wu A, Berthoux L, Grange D. Improved early results of elective hepatic resection for liver tumors: one hundred consecutive hepatectomies in cirrhotic and non cirrhotic patients. Arch Surg 1989; 124: 1033-1037.

17. Fan ST, Lo CM, Liu CL, et al. Hepatectomy for hepatocellular carcinoma: toward zero hospital deaths? Ann Surg 1999; 229: 322-330.

18. Torzilli G, Makuuchi M, Inoue K, et al. No-mortality liver resection for hepatocellular carcinoma in cirrhotic and noncirrhotic patients: is there a way? A prospective analysis of our approach. Arch Surg 1999; 134: 984-992.

19. Fan ST. Methods and related drawbacks in the estimation of surgical risks in cirrhotic patients undergoing hepatectomy. Hepatogastroenterology 2002; 49: 17-20.

20. Liu CL, Fan ST, Lo CM, Ng IO, Poon RT, Wong J. Intraoperative iatrogenic rupture of hepatocellular carcinoma. World J Surg 2002; 26: 348-352.

21. Lentschener C, Ozier Y. Anaesthesia for elective liver resection: some points should be revisited. Eur J Anaesthesiol 2002; 19: 780-788.

22. Decailliot F, Cherqui D, Leroux B, et al. Effects of portal triad clamping on haemodynamic conditions during laparoscopic liver resection. Br J Anaesth 2001; 87: 493-496. 